\title{
Dialect diversity and Chinese economy: a brief review
}

Разнообразие диалектов и китайская экономика: краткий обзор. В статье представлен предварительный анализ исследований, посвященных влиянию диалектного разнообразия на китайскую экономику. Рассматриваются три типа отношений: диалекты и трудовой капитал, диалекты и предприятия, диалекты и экономический рост на микро, мезо и макроуровне. Кроме того, поднимается вопрос влияния диалектов на общественное доверие.

The role dialects play in the Chinese economy has attracted growing attention in recent years. Following basic assumptions originated from theories of institutional economics, that languages can mould culture groups, affect both individual and organisational behaviour, and eventuality influence the market, Chinese economists have correlated various economic data to indices of dialect diversity, trying to prove those assumptions quantitatively. Their findings can be summarized on three levels.

\section{The Micro-level: Dialect and Human Capital}

On the micro-level, relations between dialects and labour migration patterns have been examined most thoroughly. One of the pioneering studies, Liu et al.(2016) noticed that in China most labor migrations occur within the same dialectal region, and put forward a generalized model that under certain conditions, dialectal distance will at first promote and then hinder labour migration, showing an inverted U-shaped pattern.

The paper created an index of dialectal distances for 278 prefectures from a family tree based on Dictionary of Chinese Dialects and Language Atlas of China (LAC), and match it to the labour migration data in China Labor-Force Dynamic Survey (CLDS 2012), and validated the model. Specifically, within the same dialectal region, if dialectal distance rises by one level, the probability of cross-prefecture migration will rise by $30 \%$, but will fall by $3 \%$ across main dialectal borders. So the optimal dialectal distance for labour migration is crossing minor dialectal areas but staying within the main dialectal region, making a good example of both the complementary effect and the identification effect. With such factors as marriage and family, social network, hukou system, geographic topography, and Mandarin usage being considered and controlled, the inverted Ushaped pattern stays robust.

Another interesting angle was provided by Jin et al.(2017), which discusses the relations between dialect and credit opportunities for the migration labor in China. The paper also employed CLDS (2012), which included 16253 surveys on individuals' education, migration, social involvements, as well as language/dialect proficiency and loan activities. The latter two sets of data were matched, showing that dialect proficiency have significantly positive impacts on credit opportunities through social capital, i.e. an implicit mechanism of trust based on cultural identification. Moreover, this effect is more significant on low-income social groups than on the highincome ones, suggesting that the latter groups incline to get loans from formal financial institutions, and the former groups from informal markets, in which social capital is essential due to the absence of mortgages. By controlling factors such as Standard Mandarin and foreign languages proficiency, conclusions remain robust.

Relations between language abilities and wages, which were discussed extensively in western literature, have also been taken into account. One of the latest studies, Ma \& Zhao (2019) found that dialectal diversity significantly decreases labour income among migrants in China's urban labour markets. Using data sets of China Household Finance Survey (CHFS) in 2011, the paper proved that when the index of dialectal diversity increases by 0.1 , the labour income will decrease by $7.52 \%$, surpassing the impact of gender. Probable mechanisms include higher transaction cost to spread knowledge and skills, and higher expenses to build individuals' social networks, both of which caused by dialectal diversity, and the paper found no obvious empirical evidence to support the former one. With factors such as geographical features, Standard Mandarin proficiency and rural/urban distinctions controlled, conclusions remain robust.

The paper also revealed some heterogeneity of the effect, that its impacts on the service sector and on women are more prominent than on industrial or agricultural sectors, and on men. Specifically, when the index of dialectal diversity increases by 0.1 , the labour income in the service sector will decrease by $9.06 \%$, and the labour income of men and women will decrease by $6.1 \%$ and $8.9 \%$ respectively. The second heterogeneity seems contrary to the common sense that women are more gifted in language learning, and this may be caused by 
samples in the data sets in which there are more women engaged in the service sector. The income itself is also relevant to the heterogeneity of the effect, that the lower the income, the stronger the impact. Groups with the income near the third quartile (richer) get only half the impact of those with the income near the first quartile (poorer).

\section{The Meso-level: Dialect and Enterprises}

On the meso-level, researchers focused on the impacts dialects impose on business management and interactions between enterprises.

Dai et al.(2016) tried to answer the question whether local accent can reduce agency cost in companies. The paper collected birthplaces of chairpersons of the board and CEOs of all A-share listed companies from 2009 to 2014 , and then matched the data to the three layers of dialectal regions dialects constructed by $L A C$, and derived some interesting findings:

(a) The companies whose chairperson and CEO come from the same dialectal region will have lower agency cost, and this effect becomes more significant when dialects are classified finer.

(b) This effect is further influenced by the nature of property, operating locations and migration. In stateowned enterprises where both chairpersons and CEOs are appointed by governments, the effect is much minor than in the private ones, and in companies operating away from hometown, the effect is also weaker comparing to those operating in hometown. In addition, due to the increasing complexity of local cultural environment and uncertainty of mutual trust caused by migration, the effect in net inflow area is stronger than in net outflow area.

As for enterprise interactions, $\mathrm{Li}$ et al.(2018) discussed the complex relations between dialects and company mergers and acquisitions. By matching dialect data from $L A C$ to the locations of acquirers and target companies listed between 2000 and 2012, the paper found that the greater the dialect distance is, the less the shareholder value is, exemplifying a typical case of how dialect or regional culture exerts influence on specific financial activities between companies.

The mechanism however, is through human capital and culture identity. As a form of corporate capital, identical dialect background can facilitate the process of company mergers and acquisitions, and enhance corporate performance afterwards.

Further analysis shows that this effect is enhanced when informal institutions play a complementary role or information asymmetry is encountered, and is more obvious in private enterprises than in state-owned enterprises.

\section{The Macro-level: Dialect and Economic Growth}

On the macro-level however, multiple studies proved the inhibiting effect of dialect diversity to regional growth and openness.

$\mathrm{Xu}$ et al.(2015) studied the correlations between dialect diversity and the amount of income/output per capita. According to The Dictionary of Chinese Dialects, which includes dialect data of 2113 counties, the paper firstly constructed an index of dialect diversity for 278 prefectures and cities, and then matched it to the their corresponding economic performances in 2010, and found significant negative effect of dialect diversity on economic growth. Other factors being constant, by completely eliminating dialect diversity, the output and income per capita can be increased by $33 \%$ and $30 \%$ respectively.

By controlling factors such as Mandarin or non-Mandarin regions, northern or southern regions and coastal or inland regions, conclusions are still robust. By controlling the factor of Standard Mandarin level, the effect remains prominent, suggesting that cultural identifications and the resulting psychological distances caused by dialects cannot be eliminated by Standard Mandarin, which is acquired through human capital investment. This in turn proves that the main mechanism that dialect diversity hinders economic development is not through obstructions in communication, but through psychological distances.

Li et al. (2017) studied the relations between dialect diversity and degrees of regional openness. By matching 276 cities' foreign trade data from 2005 to 2013 provided by China Statistical Yearbook for Regional Economy, to the index of dialect diversity constructed by Xu et al.(2015), the paper found that by increasing the dialect diversity of just one dialect, the openness degree of a city will be reduced by $2-2.4 \%$ percent, showing an obvious hindrance effect of dialect diversity. Having factors such as the city's GDP, industrial structure and distances to the main ports controlled, the conclusion remains robust.

This effect have major impact on foreign trade, but not on foreign investment, suggesting its main mechanism is through impeding the accumulation of human capital and the resulting disparity of human capital among different regions. 


\section{A Generalized Explanation: Dialect and Social Trust}

As the economic consequences of dialects being revealed and explained, some researchers thought even further, pursuing a generalized explanation on how dialects affect the economy. One of the recent studies was Huang \& Liu (2017), which correlated social trust data based on China Comprehensive Social Survey (CGSS) in 2005, to the index of dialect diversity constructed by Xu et al.(2015), and three empirically proved findings:

(a) the use of identical dialect among strangers is beneficial to establish trust, and on a large scale affect the formation of social trust;

(b) the mechanism how dialect can affect social trust is through its symbolic function of identifying people's hometown, therefore enhancing the sense of solidarity;

(c) The effect is weakened in populations of higher education levels, higher immigrant portions and regions of higher income levels. Another feature of heterogeneity lies in regional difference on dialect diversity. In eastern cities where dialect diversity is much higher than central or western cities, the effect is also more obvious.

Studies above have depicted quite a complex picture about the roles dialects play in the Chinese economy. It shows that besides its linguistic and cultural values, social and economic values of dialects are too important to be neglected. In such a culturally diversified country as China, social spheres of dialects should be elaborately planned just as Standard Mandarin, in order to balance the positive and negative effects on different levels.

\section{References}

Dai Yiyi, Xiao Jinli, and Pan Yue, 2016, Can "Local Accent" Reduce Agency Cost?--A Study Based on the Perspective of Dialects, Economic Research Journal, Vol. 12, p.p. 147-186.

Huang Jiuli \& Liu Chang, 2017, Dialect and Social Trust, Journal of Finance and Economics, Vol. 43, p.p. 83-94.

Ma Shuang and Zhao Wenbo, 2019, Linguistic Diversity and Labour Income of the Migrants-An Empirical Research Using CHFS, China Economic Quarterly, Vol.18, p.p. 393-414.

Jin Jiang, Yin Feifei and Zhang Li, 2017, Dialect and Credit Opportunities of The Migration Labor, South China Journal of Economics, Vol.9, p.p. 85-102.

Li Guangqin, Cao Jianhua, and Shao Shuai, 2017, Linguistic Diversity and Regional Openness Disparity in China, The Journal of World Economy, Vol.3, p.p. 144-168.

Li Lu, He Yuqian, and Tang Xiaoyan, 2018, Cultural Differences, Dialect Feature and M\&As, Journal of Finance and Economics, Vol.6, p.p. 140-152.

Liu Yuyun Xu Xianxiang and Xiao Zekai, 2016, The Pattern of Labor Cross-dialects Migration, Economic Research Journal, Vol. 10, 134-162.

Xu Xianxiang, Liu Yuyun, and Xiao Zekai, 2015, Dialect and Economic Growth, China Journal of Economics, Vol. 2, p.p. 1-32. 